\title{
Severe Fever with Thrombocytopenia Syndrome Associated with Manual De-Ticking of Domestic Dogs
}

\author{
Jae Keun Chung, ${ }^{1, \dagger}$ Choon Mee Kim, ${ }^{2, \dagger}$ Dong-Min Kim, ${ }^{3, *}$ Na-Ra Yun, ${ }^{3}$ Jung Wook Park, \\ Jinjong Seo, and Yang Soo Kim ${ }^{4, *}$
}

\begin{abstract}
Background: Severe fever with thrombocytopenia syndrome (SFTS) is an emerging infectious disease. SFTS is caused by the SFTS virus, a novel phlebovirus, and is spread by ticks.

Methods: A 50-year-old man was admitted to our hospital with the chief complaint of fever and was diagnosed with confirmed SFTS. An epidemiological investigation was conducted, and immunofluorescent antibody assays (IFAs) were performed to determine the role of the patient's three dogs in the transmission. PCR assays were performed using ticks that were collected with the dragging and flagging method from the patient's dogs and home. Results: PCR results were positive, and IFA confirmed an increased antibody titer. Although the reverse transcription PCR results of the three dogs were negative for SFTS virus, one dog had an elevated SFTS IFA immunoglobulin $\mathrm{G}(\mathrm{IgG})$ titer of 1:1,024. Moreover, a number of ticks were observed in the area surrounding the dog cages. Based on the findings of the patient interview, the patient was likely to have acquired SFTS by blood splash because he removed or burst ticks from the dogs with his bare hands. Although no tick bites were reported, tick transmission could not be ruled out. Studies have shown that only one in three individuals with a diagnosis of SFTS recalls a tick bite; thus, a definite exclusion of tick transmission in this case was not possible. Conclusions: The epidemiological findings of our case suggest a possible relationship between tick infestation in domestic dogs and SFTS virus transmission to humans. However, there is no direct evidence supporting this viral transmission route. Future studies are needed to further investigate a potential route of SFTS transmission by exposure to engorged tick blood or pet dogs.
\end{abstract}

Keywords: ticks, tick-borne diseases, phlebovirus, severe fever with thrombocytopenia syndrome

\section{Background}

Q EVERE FEVER WITH thrombocytopenia syndrome (SFTS) $\boldsymbol{N}$ is a tick-borne infectious disease caused by the SFTS virus (SFTSV) (Adams et al. 2017). SFTS is a disease emerging in Korea, Japan, and China (Liu et al. 2014). Although SFTSV transmission can occur through Amblyomma testudinarium and Ixodes nipponensis tick species, Haemaphysalis longicornis is the major vector of SFTSV. H. longicornis is frequently found on goats and sheep, and these animals are thus considered major intermediate hosts ( $\mathrm{Li}$ et al. 2014, Suh et al. 2016). However, SFTS-specific antibodies have also been detected in goats, cattle, pigs, and dogs (Tian et al. 2017).
In a study of 2,741 animals in Jiangsu, China, the SFTSV antibody seroprevalence was the highest in goats $(66.8 \%)$, followed by cattle $(28.2 \%)$, dogs $(7.4 \%)$, pigs $(4.7 \%)$, rodents (4.4\%), and hedgehogs (2.7\%) (Li et al. 2014). However, when enzyme-linked immunosorbent assays were performed to detect SFTSV-specific antibodies in domesticated animals in Shandong Province of China, $328(69.5 \%)$ of 472 sheep, $509(60.5 \%)$ of 842 cattle, 136 $(37.9 \%)$ of 359 dogs, $26(3.1 \%)$ of 839 pigs, and 250 $(47.4 \%)$ of 527 chickens possessed antibodies. In particular, dogs had SFTS-specific antibody of 37.9\%, and SFTS viral RNA was also detected in 19 dogs (5.3\%) (Niu et al. 2013). In Korea, PCR analyses performed in 426 dogs and 215 cats in an animal shelter confirmed the presence of the

\footnotetext{
${ }^{1}$ Health and Environment Research Institute of Gwangju Metropolitan City, Gwangju, South Korea.

${ }^{2}$ Premedical Science, College of Medicine, Chosun University, Gwangju, South Korea.

${ }^{3}$ Department of Internal Medicine, College of Medicine, Chosun University, Gwangju, South Korea.

${ }^{4}$ Department of Infectious Diseases, Asan Medical Center, University of Ulsan College of Medicine, Seoul, South Korea.

${ }^{\dagger}$ These authors contributed equally to this work.

*These authors contributed equally to this work.
} 
$\mathrm{J} 3$ clade SFTSV in $1 \mathrm{dog}(0.2 \%)$ and 1 cat $(0.5 \%)$ (Lee et al. 2017). Thus, positive results in antibody tests and RT-PCR in dogs indicate a possible SFTS transmission mediated by dogs.

Besides SFTS transmission through tick bites, humanto-human transmission caused by needle stick injury and close contact with blood and body secretions from individuals infected with SFTS has also been reported (Kim et al. 2015, Jeong et al. 2016, Yoon Park et al. 2016). However, to our knowledge, no study has investigated the role of domestic dogs in SFTS transmission, and no case of SFTS transmitted by dogs has been reported. In this study, we report a case of SFTS diagnosed in a man who owned three dogs. We describe the epidemiological investigation that aimed to determine possible SFTS transmission routes in this case.

\section{Materials and Methods}

\section{Case}

A 50-year-old Korean man presented to our hospital with the chief complaint of fever that persisted for 4 days. The patient visited the local emergency room 4 days before hospital admission and received two rounds of analgesics and fluid treatment for fever, chills, myalgia, and watery diarrhea. One day before presenting to our hospital, leukocytopenia was detected at the local emergency room, and the patient was transferred to our hospital on June 20, 2017. The patient was originally employed in the construction industry before moving to a vacation house located in a forest in a rural area in Gokseong, Jeollanam-do, in March 2017. No remarkable medical history was noted. The patient underwent a thorough physical examination at hospital admission. He reported
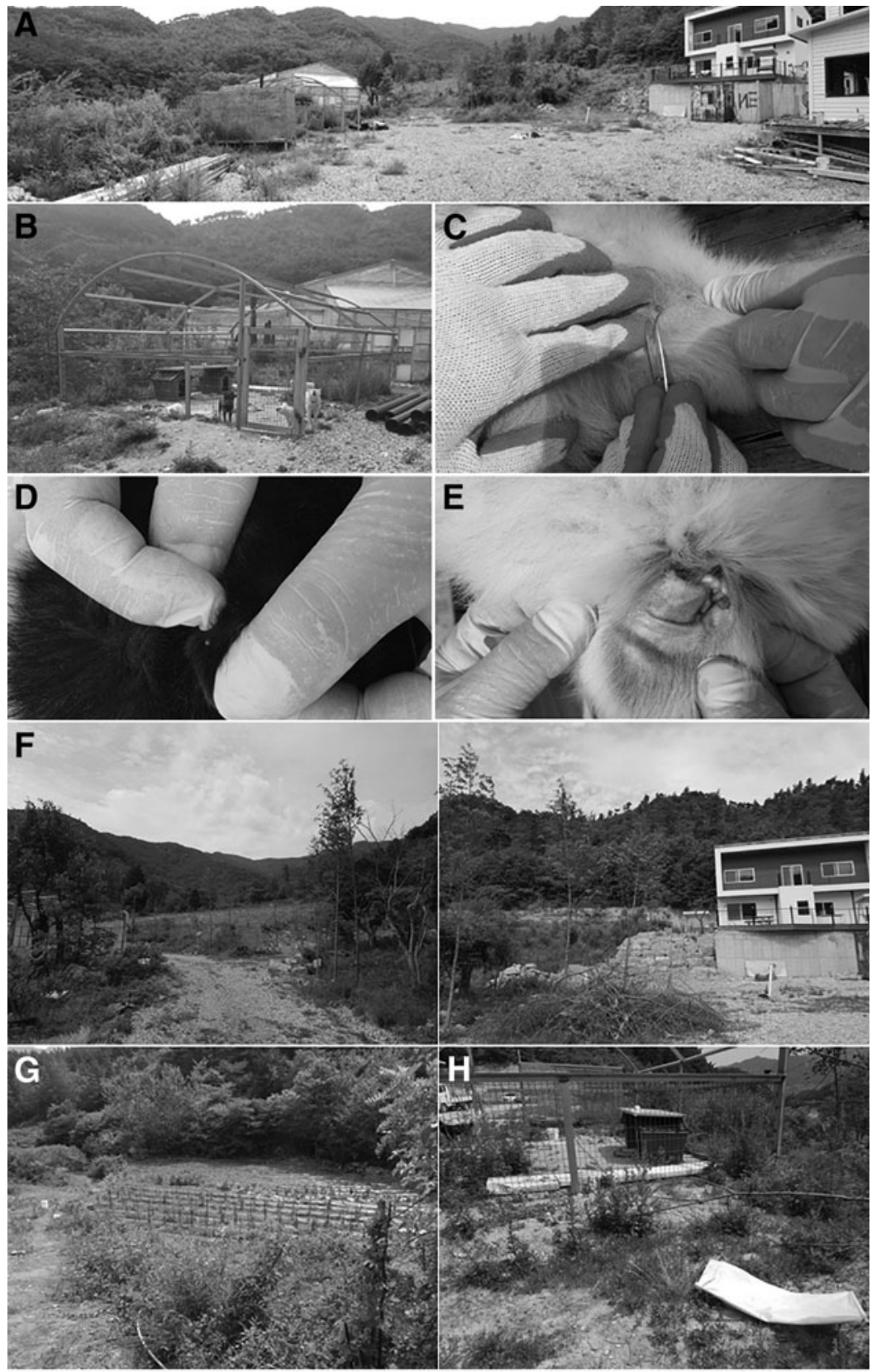

FIG. 1. First epidemiological investigation was performed in the rural vacation house and surrounding areas on June 24, 2017, the sixth day of admission. The patient, a 50year-old man, presented to our hospital with the chief complaint of fever and was confirmed to have SFTS. (A) The house to which the patient moved recently. The house is located on the right, and the dog cages can be seen on the left. (B) Outdoor dog cages, with dog B (black 8-month-old female Jindo) on the far left, dog C (white 3-month-old male Pungsan) in the middle, and $\operatorname{dog} \mathrm{A}$ (white 10-month-old female Pungsan) on the far right. (C) Ticks confirmed in the axillary area of dog A (10-month-old female Pungsan). (D) Ticks confirmed on the body of dog B (8-month-old female Jindo). (E) Ticks confirmed on the ear of dog C (3-month-old male Pungsan). (F) The patient's house and the hills and forest located to the right of the house (12 Haemaphysalis longicornis nymphs were collected over a period of $30 \mathrm{~min}$ ). (G) Corn field near the patient's house (one adult female and two $H$. longicornis nymphs were collected over a period of $30 \mathrm{~min}$ ). (H) Dog cages and surrounding areas (28 nymphs and 1 female adult $H$. longicornis were collected over a period of $30 \mathrm{~min}$ ). SFTS, severe fever with thrombocytopenia syndrome. 
having never experienced any tick bites, and no suspected site of a tick bite was observed. The patient owned three dogs.

At the time of admission, the patient's blood pressure was $120 / 70 \mathrm{mmHg}$; pulse, 86 beats $/ \mathrm{min}$; respiratory rate, 20 breaths/min; and body temperature, $39.3^{\circ} \mathrm{C}$. The results of the blood test performed at admission were as follows: white blood cell count, 2,040 (neutrophils 59.7\%; normal range, 4,80010,800) cells $/ \mu \mathrm{L}$; hemoglobin, 14.6 (normal range, 12-18) g/dL; and platelets, 97,000 (normal range, 130,000-450,000) cells $/ \mu \mathrm{L}$. The results of the biochemistry test were as follows: aspartate aminotransferase, 71.9 (reference range, 5-40) IU/L; alanine aminotransferase, 26.3 (reference range, 5-40) IU/L; total bilirubin, 0.4 (reference range, $0.2-1.2$ ) $\mathrm{mg} / \mathrm{dL}$; blood urea nitrogen, 17.6 (reference range, 8.0-20) $\mathrm{mg} / \mathrm{dL}$; and creatinine, 1.02 (reference range, $0.5-1.3) \mathrm{mg} / \mathrm{dL}$. Although the erythrocyte sedimentation rate was 10 (reference range, $0-15) \mathrm{mm} / \mathrm{h}$, the C-reactive protein level was 0.11 (reference range, $0.0-0.3$ ) $\mathrm{mg} / \mathrm{dL}$, and the following parameters had elevated values: lactate dehydrogenase, 471 (reference range, 200-450) U/L; creatine phosphokinase, 1,445 (reference range, 55-215) U/L; creatine phosphokinase-MB, 7.80 (reference range, 0-6) $\mathrm{ng} / \mathrm{mL}$; and ferritin, 569 (reference range, 21.8-274.6) ng/mL. PCR assays and immunofluorescent antibody assays (IFAs) for vector-borne infectious diseases, including scrub typhus, spotted fever, anaplasmosis, and Lyme disease, yielded negative results (Fenollar et al. 2004, Klempa et al. 2006, Takano et al. 2009, Kato et al. 2013, Sapi et al. 2013). However, because the SFTSV-specific L (encoding RNA-dependent RNA polymerase)-, S (encoding viral nucleocapsid and nonstructural proteins)-, and M (encoding viral envelope glycoprotein)segment targeting RT-PCR and RT nested-PCR (RT N-PCR), as well as the S-segment targeting real-time RT-PCR results were positive, conservative treatment (such as acetaminophen and fluid therapy) was initiated (Zhang et al. 2012, Yun et al. 2014, Yoshikawa et al. 2015). The patient received 1 week of treatment, after which he showed an improvement in symptoms and was discharged.

\section{Epidemiological investigation}

We visited the patient's home to determine the route of infection. The patient built a vacation house located in a forest in a rural part of Gokseong in March 2017 and lived there since May 2017. Dog cages surrounded by fences $\left(16 \mathrm{~m}^{2}\right)$ were found in front of the two-story house (Fig. 1A). At the time of admission to our hospital, the patient lived with his wife and three $\operatorname{dogs}$ : $\operatorname{dog}$ A (10-month-old female Pungsan), dog B (8-month-old female Jindo), and dog C (3month-old male Pungsan) (Fig. 1B). The three dogs wandered in the mountains surrounding the house almost every day and sometimes returned with badgers or elks that they hunted. Dog A in particular was known to be a hunter. According to the patient interview, the patient did not work on his farm himself and instead hired people to do the farming work whenever necessary. Over the past 3 months, the patient had been removing ticks from the dogs' fur and bursting them with his bare hand. Although the patient and his wife reported spraying the dogs' bodies with a pesticide for ticks, 2-Sec-butylphenyl-N-methylcarbamate (Barasal$\mathrm{P}^{\circledR}$; Shinna Medicine, Daegu, Korea), five ticks from dog A (Fig. 1C), six ticks from dog B (Fig. 1D), and seven ticks from $\operatorname{dog} C$ (Fig. 1E) were collected at the first visit on June
24, 2017. Moreover, ticks were collected using the dragging and flagging method for $30 \mathrm{~min}$ at each of the following locations: in the hills and forests around the house (Fig. 1F), in the corn field near the house (Fig. 1G), and around the dog cages (Fig. 1H). At the second visit on August 12, 2017, more ticks were collected from the same three sites (Fig. 2A), and 3, 4, and 12 ticks were removed from dog A, $\operatorname{dog} B$, and $\operatorname{dog} C$, respectively. However, all dogs seemed healthy; in particular, dog A gave birth to eight healthy pups on the day of our second visit for the epidemiological investigation (Fig. 2B).

\section{SFTS diagnosis}

The collected ticks were washed in $70 \%$ ethanol for 2-5 min and then washed thrice in sterile triple-distilled water and gently dried. Next, the samples were pulverized in tubes containing 2.8-mm stainless steel beads with $800 \mu \mathrm{L}$ of phosphate-buffered saline (PBS; $10 \%$ fetal bovine serum and $5 \%$ penicillin/streptomycin), followed by grinding for $20 \mathrm{~s}$ at 4,800 rpm using a BioSpec 3110BX Mini-Beadbeater-1 high energy cell disrupter (BioSpec).
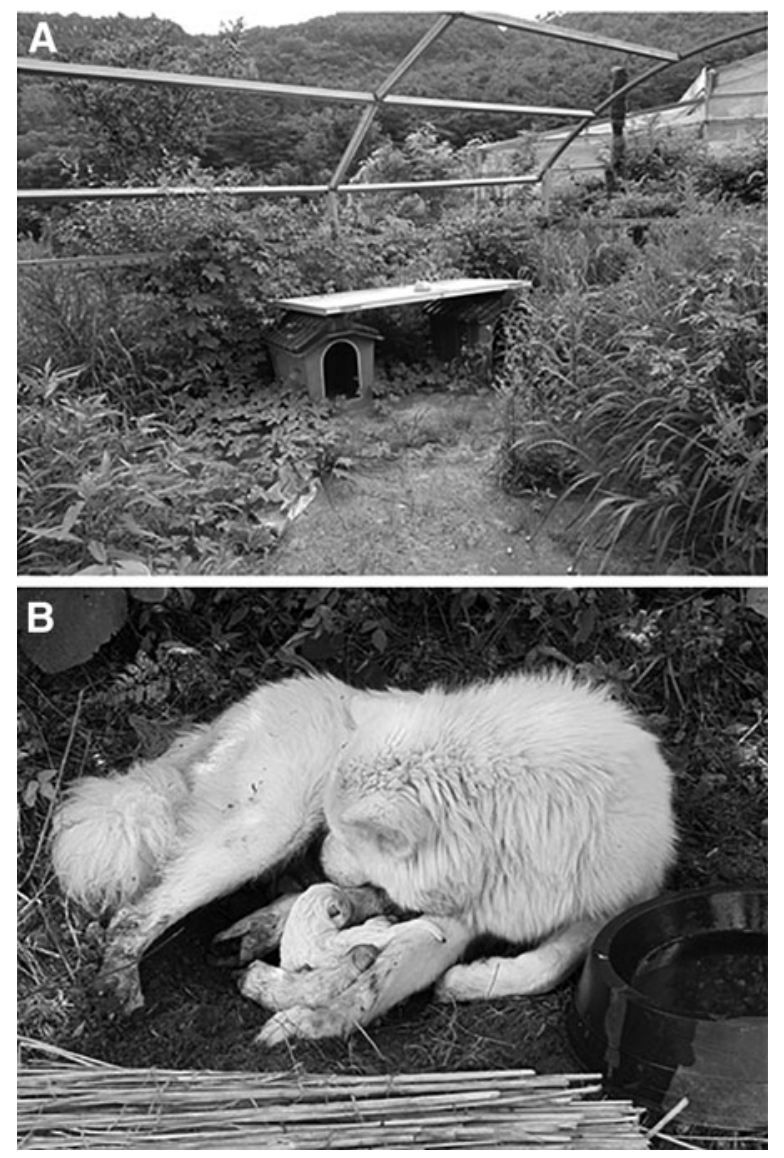

FIG. 2. (A) Dog cages and surrounding areas observed during the second epidemiological investigation (August 12, 2017 ) of the patient's home. (1 adult, 1 nymph, and 48 larvae $H$. longicornis were collected over a period of $30 \mathrm{~min}$ from the areas surrounding the dog cages). (B) Dog A gave birth during the second visit on August 12, 2017. The dog and all pups were healthy. 


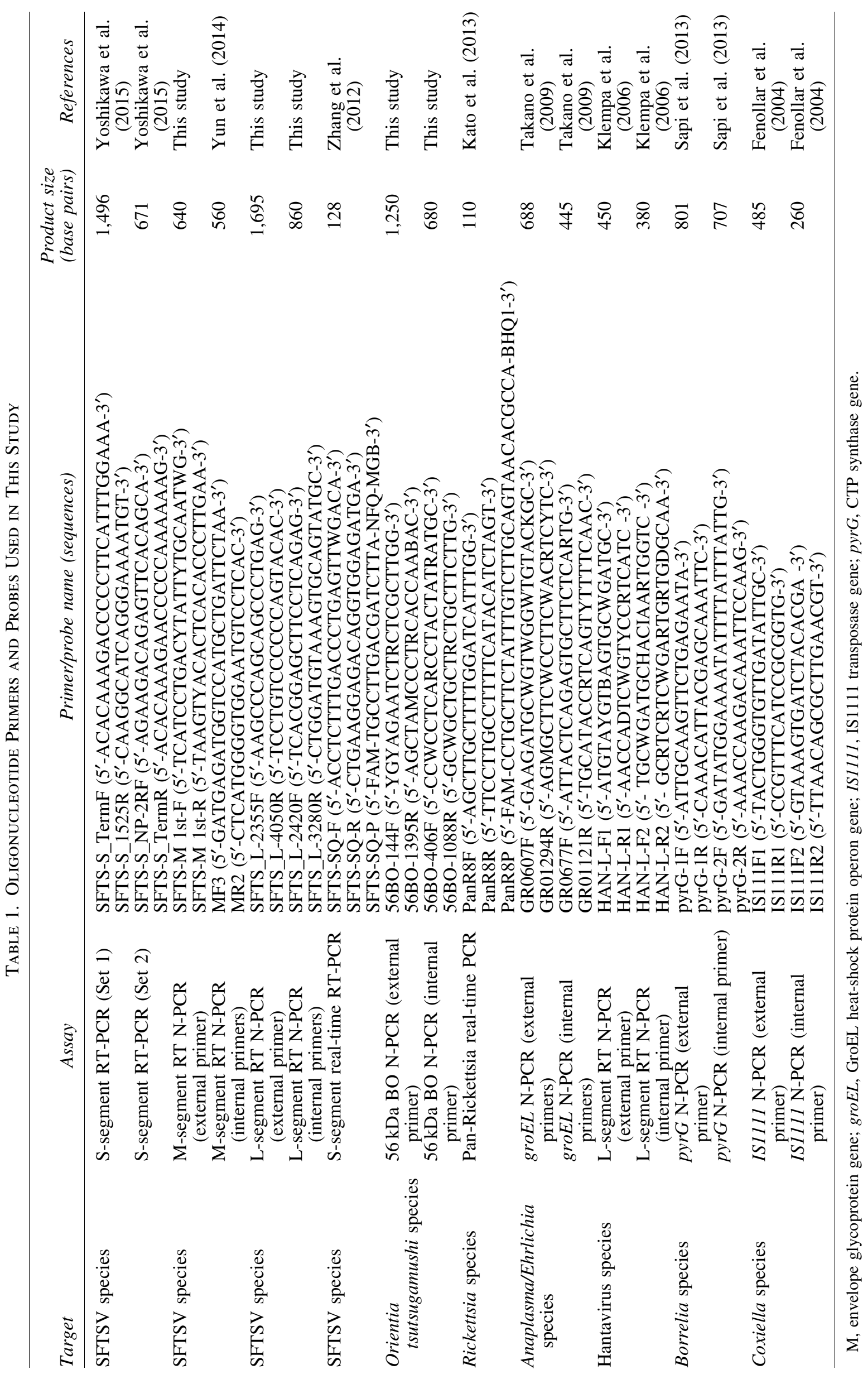


At the time of admission, viral RNA was extracted from the patient's plasma, which was analyzed using a Viral Gene$\operatorname{spin}^{\mathrm{TM}}$ Viral DNA/RNA Extraction Kit (iNTRON Biotechnology, Sungnam-si, Korea). SFTSV-targeted RT-PCR and RT N-PCR were performed using complementary DNA (cDNA) synthesized with the SuperScript VILO MasterMix (Invitrogen), AmpliTaq Gold 360 Master Mix (Applied Biosystems), S-, M-, and L-segment specific PCR primers, and Veriti ${ }^{\mathrm{TM}}$ 96Well thermal cycler (Applied Biosystems) (Yun et al. 2014, Yoshikawa et al. 2015). For RT-PCR, $20 \mu \mathrm{L}$ of reaction solution was used; this solution comprised $1 \mu \mathrm{L}$ each of first PCR primers $(5 \mu \mathrm{M}), 10 \mu \mathrm{L}$ of Master Mix, $2 \mu \mathrm{L}$ of GC enhancer, $4 \mu \mathrm{L}$ of distilled water, and $2 \mu \mathrm{L}$ of cDNA as template DNA. The same reaction mixture used for RT-PCR was used for RT N-PCR, except the first PCR product was used as template DNA and the N-PCR primers were included. For SFTS realtime RT-PCR, previously reported primers and probe targeting the S-segment were used (Zhang et al. 2012). The PCR primers and probes used in this study are listed in Table 1.

For IFA against the virus, Vero E6 cells infected with SFTSV were inoculated and fixed with acetone on Tefloncoated well slides to prepare the SFTS antigen slide. The serum was diluted with a twofold serial dilution and then reacted with viral antigens in a moist chamber for $30 \mathrm{~min}$ at $37^{\circ} \mathrm{C}$. After washing with PBS and distilled water, fluorescein isothiocyanate (FITC)-conjugated anti-human immunoglobulin $\mathrm{G}(\mathrm{IgG})$ and immunoglobulin $\mathrm{M}(\mathrm{IgM})$ as secondary antibodies (MP Biomedicals) were applied to each slide and incubated at $37^{\circ} \mathrm{C}$ in a humid chamber for $30 \mathrm{~min}$. Subsequently, the slides were washed and dried as described above and observed under a fluorescent microscope under $\times 400$ magnification. The final serum dilution, which indicates the specific fluorescence, was used to determine the antibody titer (Park et al. 2014). RT-PCR was also performed as described above using blood collected from the three dogs. To detect IgG and IgM titers against the SFTSV, anti-dog IgG (whole molecule)-FITC antibodies produced in rabbit (Sigma) were used as the SFTS secondary antibody.

\section{Results}

No IgG or IgM was detected by the SFTS virus IFA performed with the serum collected from the patient at admission. However, in a follow-up test performed 2 weeks later, IgM and IgG titers showed more than fourfold elevations (1:128 for IgM and 1:512 for IgG; Table 2). The SFTSV M- and Lsegment targeting RT N-PCR and two sets of S-segment targeting RT-PCR were performed using the patient's plasma, and each amplicon was cloned to determine its sequence. The determined sequence was analyzed using the BlastN (Bethesda, MD) program from the National Center for Biotechnology Information (National Institutes of Health). In gene sequencing analysis of SFTSV detected in the patient's plasma, the S-segment sequences from the two-set RT-PCR and the M- and L-segment sequences from RT N-PCR showed $99.2 \%, 99.6 \%$, and $99.5 \%$ sequence similarity, respectively, with the same SFTS strain KAJNH2 (accession no. KJ739588), which was previously detected in another Korean patient. A phylogenetic tree analysis was performed based on the S-, M-, and L-segment sequences from the patient and from various SFTSV strains in GenBank (Fig. 3A-C); the SFTSV could be grouped with the SFTSV collected from the blood of

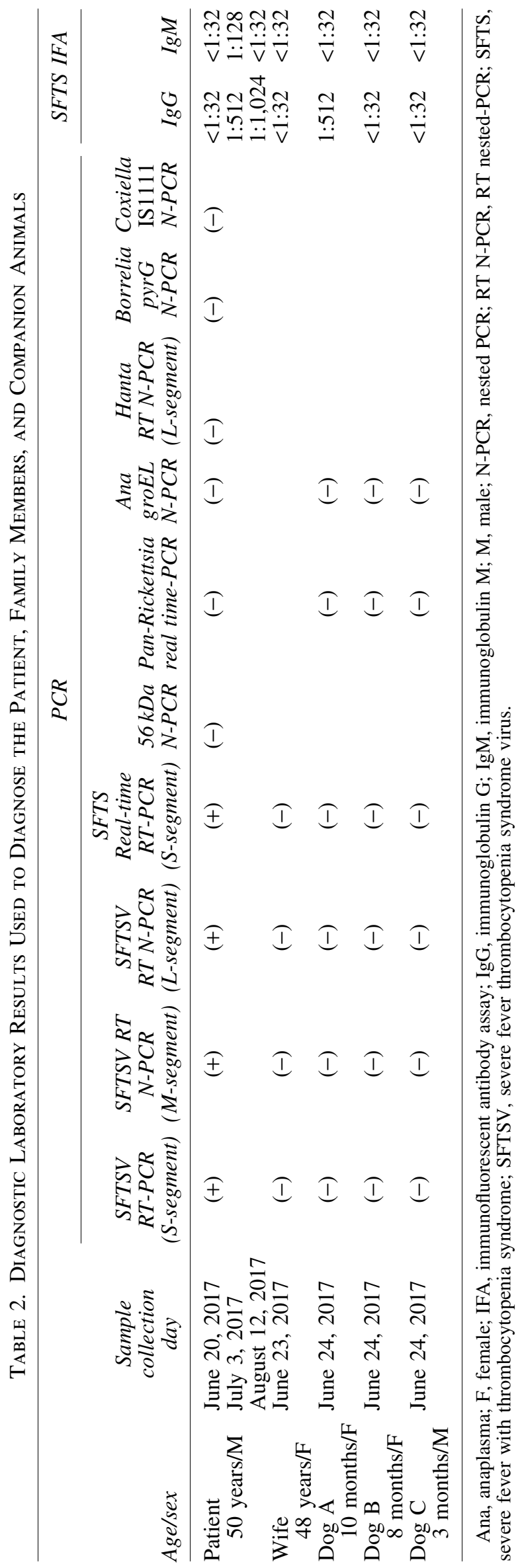




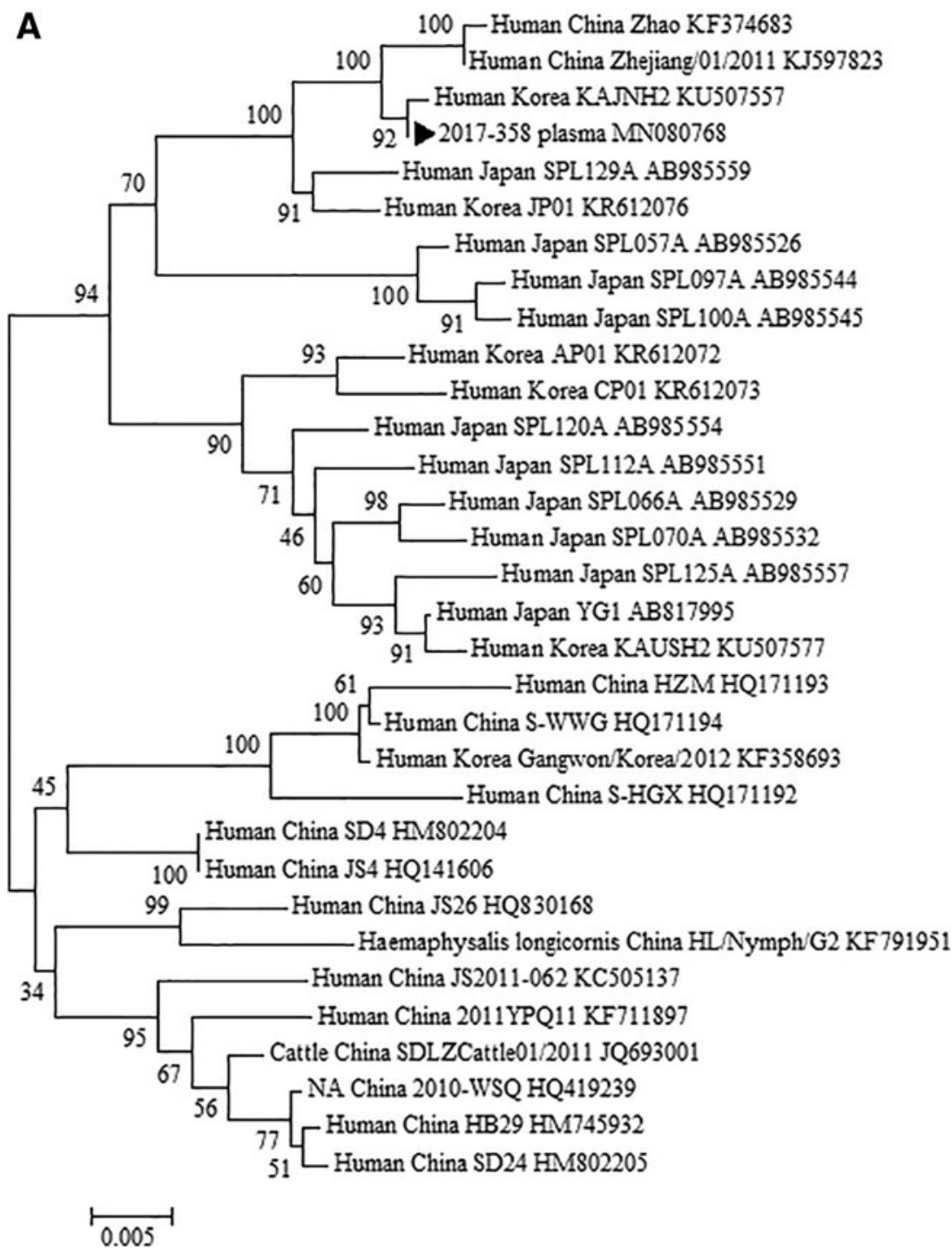

FIG. 3. A phylogenetic tree based on the SFTSV S-segment (A, 738 bp), Msegment (B, $477 \mathrm{bp})$, and L-segment (C, 696 bp) partial sequences from GenBank and the specimen collected in this study ( ) . Scale bars indicate $0.005(\mathbf{A}, \mathbf{C})$ and 0.01 (B) base substitutions per site.

a Korean patient with SFTS. The phylogenetic tree generated with SFTSV S-, M-, and L-segment sequences showed similar topologies. SFTSV RT-PCR performed using the same sample showed a cycle threshold $(\mathrm{Ct})$ value of 38.32. No SFTSspecific antibody was detected in the SFTS IFA performed using the blood of the patient's wife; the PCR test also yielded negative results. In the SFTS IFA performed using the blood of the three dogs, the test yielded positive results for only the serum of $\operatorname{dog}$ A (IgG 1:1,024); however, no SFTS-specific antibodies were detected in $\operatorname{dog} \mathrm{B}$ or $\operatorname{dog} \mathrm{C}$.

Although we expected to isolate the SFTSV in the blood of the dogs collected in the first epidemiological investigation, the SFTSV targeting RT-PCR assay yielded negative results. When the ticks removed from the dogs in the first epidemiological investigation were observed under a microscope, all five ticks from $\operatorname{dog} \mathrm{A}$, six from $\operatorname{dog} \mathrm{B}$, and seven from $\operatorname{dog} \mathrm{C}$ were confirmed as $H$. longicornis. When the ticks were collected at each of the 3 locations around the patient's house, 28 nymphs and 1 female adult $H$. longicornis were found around the dog cages (Fig. 1H). In the corn field (Fig. 1G), 1 female adult and $2 \mathrm{H}$. longicornis nymphs were detected, and $12 \mathrm{H}$. longicornis nymphs were detected in the hills and forest around the house (Fig. 1F).

When the collection procedure was performed at the same locations during the second investigation, 1 adult, 1 nymph, and 48 larvae were collected around the dog cages (Fig. 2A), while 4 nymphs were collected in the hills and forest and none was found in the corn field. All were confirmed to be H. longicornis. Moreover, the 3 ticks from $\operatorname{dog}$ A, 4 from dog $\mathrm{B}$, and 12 from $\operatorname{dog} \mathrm{C}$ were all confirmed to be $H$. longicornis. Although the SFTSV-targeting RT-PCR assay was performed using the $H$. longicornis that were removed from the dogs and collected from the three locations during both investigations, no SFTSV was detected.

\section{Discussion}

When the Japanese Health Ministry reported the death of a Japanese woman due to SFTS transmitted by a cat bite in 2017, the possibility of mammal to human transmission of SFTS became an important social issue (Hwang et al. 2017). Following that report, an RT-PCR assay was performed on 


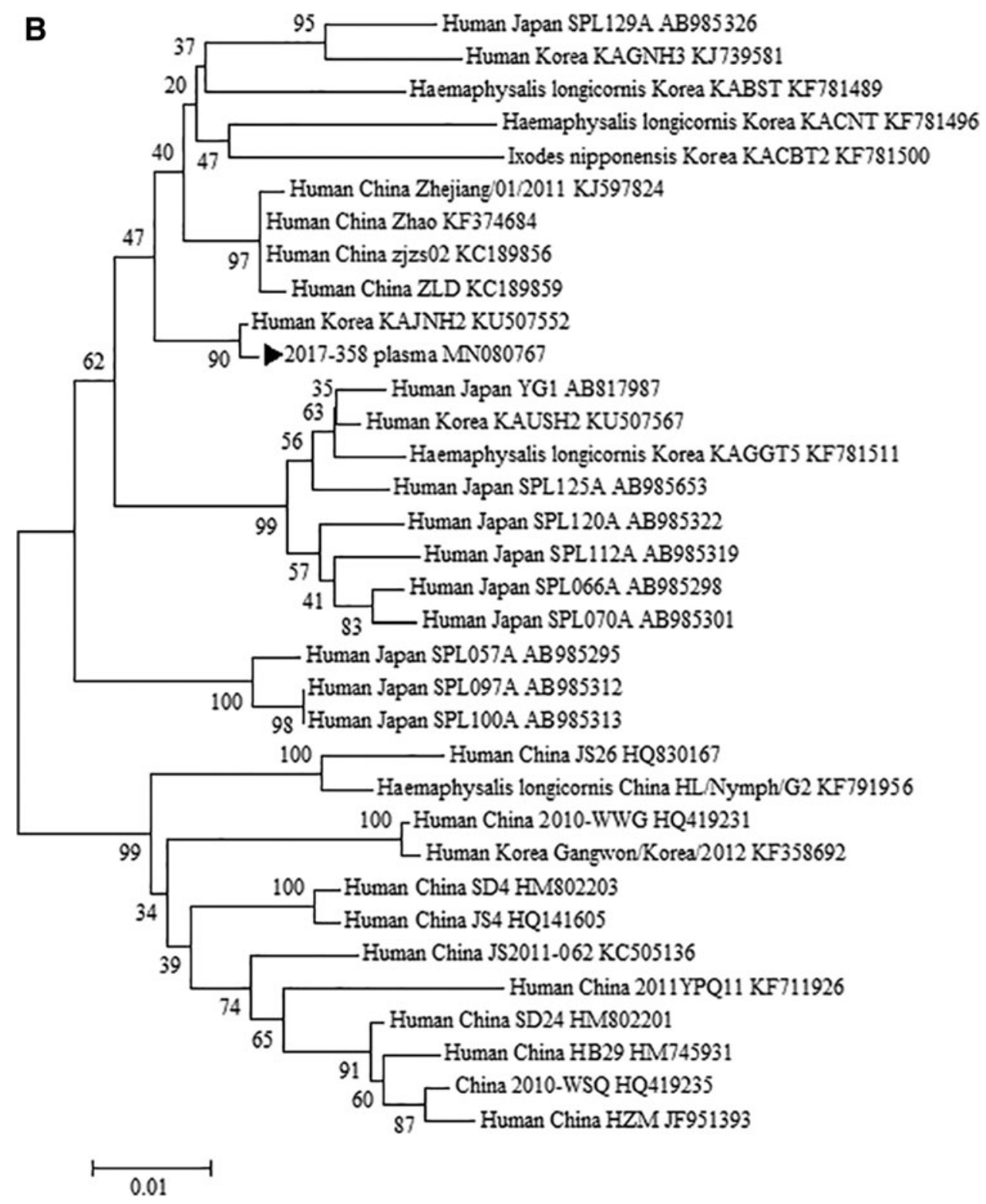

FIG. 3. Continued

(continued)

126 feral cats in Seoul, South Korea, and the SFTSV was detected in $22(17.5 \%)$ of these cats (Hwang et al. 2017). Although companion animals living in close proximity to humans can provide humans with positive benefits such as emotional support, there can also be negative aspects, including the potential of zoonotic disease transmission (Day 2016). Dogs are the commonest companion animals worldwide and can be infested with fleas and ticks, which are then transported into the peridomestic environment. Such vectors can bite a human host, thereby transmitting an infectious disease, and can also act as a reservoir of vector-borne infectious diseases. Veterinarians in Korea promote the use of preventive strategies to avoid these infestations.

The seroprevalence of antibodies against Borrelia burgdorferi in dogs was a sensitive and reliable measure of the potential risk of $B$. burgdorferi infection in humans (Lindenmayer et al. 1991, Beard 2014). Paddock also addresses several possible transmission routes in the context of Rickettsia rickettsii transmission from a dog to its owner, including manual de-ticking. Both dogs and humans can acquire Rocky Mountain spotted fever (and many other ticktransmitted pathogens) from ticks, potentially resulting in a fatal illness in both species (Paddock et al. 2002). However, no case of dog-mediated SFTS transmission has yet been reported. Furthermore, no study has investigated the role of dogs in SFTS transmission, with no reported evidence of illness in dogs infected with SFTS. However, we found two recent reports on SFTS infection in dogs; both reports found that the dogs had no significant clinical signs associated with SFTS (Cheng et al. 2019, Kang et al. 2019).

The major mode of transmission of SFTS involves bites by ticks infected with the SFTSV; however, cases of humanto-human transmission due to close contact with blood or bodily secretions of patients with SFTS have been reported 


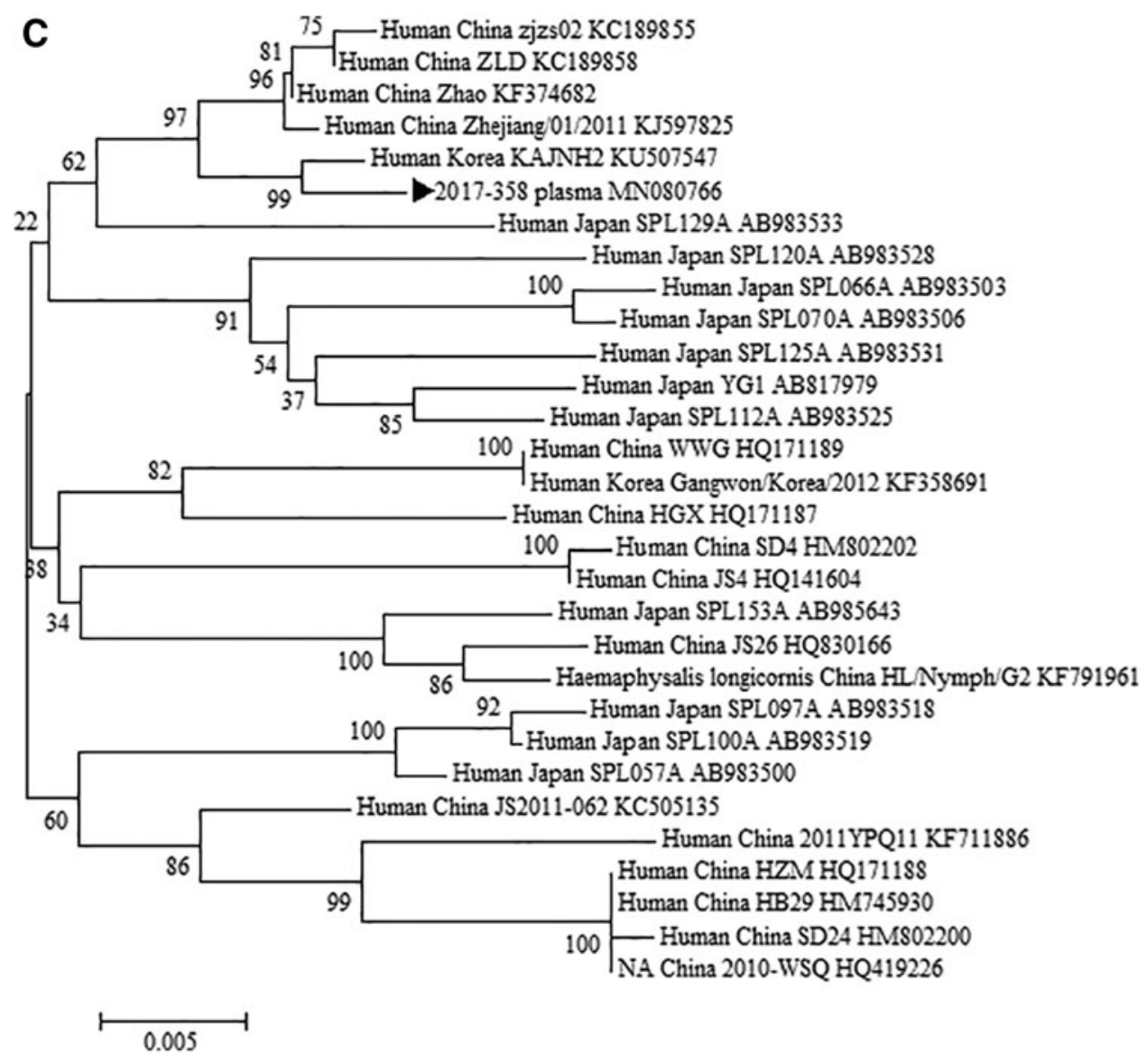

FIG. 3. Continued

(Jeong et al. 2016). Nosocomial transmission has also been reported in emergency departments and intensive care unit settings, (Kim et al. 2015) as well as transmission due to needle stick injury (Yoon Park et al. 2016). However, there are no previous reports of possible SFTS transmission caused by blood splash during removing or bursting ticks, as in our case.

In our case, the patient hired other people to work in his field rather than working on his farm himself, and thus the possibility that the patient was bitten by a tick while working outside is low. A mere $29 \%$ of patients with SFTS experienced a tick bite within the 2 weeks before disease onset (Sun et al. 2014), although lack of tick bite history or a visible bite mark does not eliminate the possibility of a tick bite. In our case, the three dogs owned by the patient were free to explore the forests around the house every day. It is likely that one or more of the dogs were bit by a tick infected with the SFTSV, while exploring the forests. This is also suggested by the SFTS antibodies identified in dog A that hunts actively. The patient also reported removing ticks from the dogs while they lay across his legs using his bare hands and wearing no protective equipment. Thus, SFTS could have been transmitted from the dogs to the patient by blood splash when the patient burst the ticks with his hands. However, further study is needed to clarify the possible mechanism by which bursting a tick could lead to SFTS transmission, such as intraocular, intraoral, or blood to blood. When we performed SFTSV-targeting RT-PCR assay with the H. longicornis removed from the three dogs, no SFTSV was detected.

In 2016, Oh et al investigated 891 ticks collected from 65 wild animals in Korea, and reported an average minimum infection rate of SFTSV in H. longicornis, Haemaphysalis flava, and I. nipponensis of $4.51 \%, 2.22 \%$, and $22.73 \%$, respectively (Oh et al. 2016). According to a report on ticks from vegetation in five national parks of Korea, the SFTSpositive rates in ticks were $4.77 \%(48 / 1,006)$ in $H$. longicornis, $1.15 \%(5 / 436)$ in $H$. flava, $0 \%(0 / 23)$ in I. nipponensis, and $20 \%$ (1/5) in A. testudinarium (Jo et al. 2019). The epidemiological investigation of our report collected 135 ticks from the area around the dog cages, and none of the ticks was positive for SFTSV. However, the high prevalence of ticks around areas such as outdoor dog cages suggests that care must be taken to prevent tick bites, especially by those with domestic animals.

\section{Conclusions}

These findings suggest a possible relationship between dogs that carry ticks and SFTS viral transmission to humans. However, there is no direct evidence supporting this route of viral transmission. Future studies are needed to clarify the potential route of SFTS transmission through pet animals. 


\section{Ethics Approval and Consent to Participate}

This study was approved by IRB of Chosun University. A written consent to participate in this study was obtained from patient. Informed consent for using client-owned animals in this study was obtained from the client or owner of the animals and experiments were performed adhering to a high standard (best practice) of veterinary care.

\section{Consent for Publication}

Written informed consent for publication of their clinical details and/or clinical images was obtained from the patient.

\section{Authors' Contributions}

J.K.C. and C.M.K. collected the patient's clinical data, wrote the article, and revised the draft during the course of submission. D.-M.K. designed and coordinated the study and contributed to drafting and reviewing the article during the course of submission. N.-R.Y. supervised the clinical case interpretation and revised the article. J.W.P., J.S., and Y.S.K. helped to draft the article. All the authors read and approved the final version of the article.

\section{Author Disclosure Statement}

No competing financial interests exist.

\section{Funding Information}

This research was supported by a grant from the Korea Health Technology R\&D Project through the Korea Health Industry Development Institute (KHIDI), funded by the Ministry of Health \& Welfare, Republic of Korea (grant number: HI15C2891). There is no role of the funding body in the design of the study, collection, analysis, and interpretation of the data.

\section{References}

Adams MJ, Lefkowitz EJ, King AM, Harrach B, et al. Changes to taxonomy and the International Code of Virus Classification and Nomenclature ratified by the International Committee on Taxonomy of Viruses (2017). Arch Virol 2017; 162:2505-2538.

Beard CB. Lyme disease prevention and control-the way forward. Can Commun Dis Rep 2014; 40:91-94.

Cheng J, Zhang L, Hu B, Wang Q, et al. Prevalence and molecular phylogenetic analysis of severe fever with thrombocytopenia syndrome virus in domestic animals and rodents in Hubei Province, China. Virol Sin 2019; 34:596600.

Day MJ. Pet-related infections. Am Fam Physician 2016; 94 : 794-802.

Fenollar F, Fournier PE, Raoult D. Molecular detection of Coxiella burnetii in the sera of patients with $\mathrm{Q}$ fever endocarditis or vascular infection. J Clin Microbiol 2004; 42: 4919-4924.

Hwang J, Kang JG, Oh SS, Chae JB, et al. Molecular detection of severe fever with thrombocytopenia syndrome virus (SFTSV) in feral cats from Seoul, Korea. Ticks Tick Borne Dis 2017; 8:9-12.
Jeong EJ, Song JY, Lim CS, Lee I, et al. Viral shedding from diverse body fluids in a patient with severe fever with thrombocytopenia syndrome. J Clin Virol 2016; 80: 33-35.

Jo YS, Kang JG, Chae JB, Cho YK, et al. Prevalence of severe fever with thrombocytopenia syndrome virus in ticks collected from national parks in Korea. Vector Borne Zoonotic Dis 2019; 19:284-289.

Kang JG, Cho YK, Jo YS, Chae JB, et al. Severe fever with thrombocytopenia syndrome virus in dogs, South Korea. Emerg Infect Dis 2019; 25:376-378.

Kato CY, Chung IH, Robinson LK, Austin AL, et al. Assessment of real-time PCR assay for detection of Rickettsia spp. and Rickettsia rickettsii in banked clinical samples. J Clin Microbiol 2013; 51:314-317.

Kim WY, Choi W, Park SW, Wang EB, et al. Nosocomial transmission of severe fever with thrombocytopenia syndrome in Korea. Clin Infect Dis 2015; 60:1681-1683.

Klempa B, Fichet-Calvet E, Lecompte E, Auste B, et al. Hantavirus in African wood mouse, Guinea. Emerg Infect Dis 2006; 12:838-840.

Lee SH, Kim HJ, Byun JW, Lee MJ, et al. Molecular detection and phylogenetic analysis of severe fever with thrombocytopenia syndrome virus in shelter dogs and cats in the Republic of Korea. Ticks Tick Borne Dis 2017; 8:626-630.

Li Z, Hu J, Bao C, Li P, et al. Seroprevalence of antibodies against SFTS virus infection in farmers and animals, Jiangsu, China. J Clin Virol 2014; 60:185-189.

Lindenmayer JM, Marshall D, Onderdonk AB. Dogs as sentinels for Lyme disease in Massachusetts. Am J Public Health 1991; 81:1448-1455.

Liu Q, He B, Huang SY, Wei F, et al. Severe fever with thrombocytopenia syndrome, an emerging tick-borne zoonosis. Lancet Infect Dis 2014; 14:763-772.

Niu G, Li J, Liang M, Jiang X, et al. Severe fever with thrombocytopenia syndrome virus among domesticated animals, China. Emerg Infect Dis 2013; 19:756-763.

Oh SS, Chae JB, Kang JG, Kim HC, et al. Detection of severe fever with thrombocytopenia syndrome virus from wild animals and Ixodidae ticks in the Republic of Korea. Vector Borne Zoonotic Dis 2016; 16:408-414.

Paddock CD, Brenner O, Vaid C, Boyd DB, et al. Short report: concurrent Rocky Mountain spotted fever in a dog and its owner. Am J Trop Med Hyg 2002; 66:197-199.

Park SW, Han MG, Yun SM, Park C, et al. J. Severe fever with thrombocytopenia syndrome virus, South Korea, 2013. Emerg Infect Dis 2014; 20:1880-1882.

Sapi E, Pabbati N, Datar A, Davies EM, et al. Improved culture conditions for the growth and detection of Borrelia from human serum. Int J Med Sci 2013; 10:362-376.

Suh JH, Kim HC, Yun SM, Lim JW, et al. Detection of SFTS virus in Ixodes nipponensis and Amblyomma testudinarium (Ixodida: Ixodidae) collected from reptiles in the Republic of Korea. J Med Entomol 2016; 53:584-590.

Sun J, Chai C, Lv H, Lin J, et al. Epidemiological characteristics of severe fever with thrombocytopenia syndrome in Zhejiang Province, China. Int J Infect Dis 2014; 25 : 180-185.

Takano A, Ando S, Kishimoto T, Fujita H, et al. Presence of a novel Ehrlichia sp. in Ixodes granulatus found in Okinawa, Japan. Microbiol Immunol 2009; 53:101-106.

Tian H, Yu P, Chowell G, Li S, et al. Severe fever with thrombocytopenia syndrome virus in humans, domesticated 
animals, ticks, and mosquitoes, Shaanxi Province, China. Am J Trop Med Hyg 2017; 96:1346-1349.

Yoon Park S, Kim SH, Park SW, Wang EB, et al. Needle-stick injury caused by a patient with severe fever with thrombocytopenia syndrome in Korea. Infect Control Hosp Epidemiol 2016; 37:368-369.

Yoshikawa T, Shimojima M, Fukushi S, Tani H, et al. Phylogenetic and geographic relationships of severe fever with thrombocytopenia syndrome virus in China, South Korea, and Japan. J Infect Dis 2015; 212:889-898.

Yun SM, Lee WG, Ryou J, Yang SC, et al. Severe fever with thrombocytopenia syndrome virus in ticks collected from humans, South Korea, 2013. Emerg Infect Dis 2014; 20: 1358-1361.
Zhang YZ, He YW, Dai YA, Xiong Y, et al. Hemorrhagic fever caused by a novel Bunyavirus in China: pathogenesis and correlates of fatal outcome. Clin Infect Dis 2012; 54:527-533.

Address correspondence to: Dong-Min Kim

Department of Internal Medicine College of Medicine Chosun University 588 Seosuk-Dong, Dong-gu Gwangju 501-717 South Korea

E-mail: drongkim@chosun.ac.kr 